Open Access

\title{
The rat placental renin-angiotensin system - a gestational gene expression study
}

Kanchan Vaswani ${ }^{1}$, Hsiu-Wen Chan ${ }^{1}$, Pali Verma ${ }^{1}$, Marloes Dekker Nitert ${ }^{1}$, Hassendrini N. Peiris ${ }^{1}$, Ryan J. Wood-Bradley 2,3 , James A. Armitage ${ }^{2,3}$, Gregory E. Rice ${ }^{1}$ and Murray D. Mitchell ${ }^{1 *}$

\begin{abstract}
Background: The placenta is an essential organ that provides nutrients and oxygen to the developing fetus and removes toxic waste products from the fetal circulation. Maintaining placental blood osmotic pressure and blood flow is crucial for viable offspring. The renin-angiotensin system (RAS) in the placenta is a key player in the regulation of maternal-fetal blood flow during pregnancy. Therefore, the aim of this study was to determine if RAS genes are differentially expressed in mid to late gestation in rat placenta.

Methods: Whole placental tissue samples from pregnant Sprague Dawley rats at embryonic (E) days 14.25, 15.25, 17.25 and 20 ( $n=6$ for each gestational age) were used for genome-wide gene expression by microarray. RAS genes with expression differences of $>2$ fold were further analyzed. Quantitative Real-Time PCR (qPCR) was performed on independent samples to confirm and validate microarray data. Immunohistochemisty and Western blotting were performed on a differentially expressed novel RAS pathway gene (ANPEP).

Results: Six out of 17 genes of the RAS pathway were differentially expressed at different gestational ages. Gene expression of four genes (Angiotensin converting enzyme (Ace), angiotensin converting enzyme 2 (Ace2),

membrane metalloendopeptidase (Mme) and angiotensin II receptor 1A (Agtr1a)) were significantly upregulated at E20 whereas two others (Thimet oligopeptidase 1 (Thop1) and Alanyl aminopeptidase (Anpep)) were downregulated at E20 prior to the onset of labour. These changes were confirmed by qPCR. Western blots revealed no overall differences in ANPEP protein expression in the placentae. Immunohistochemical studies, however, indicated that the localization of ANPEP differed at E17.25 and E20 as ANPEP localization in the giant trophoblast cell of the junctional zone was no longer detectable at E20.

Conclusions: The current study investigated the expression of members of the RAS pathway in rat placentae and observed significantly altered expression of 6 RAS genes at 4 gestational ages. These findings present the need for further comprehensive investigation of RAS genes in normal and complicated pregnancies.
\end{abstract}

Keywords: Placenta, Renin-Angiotensin System, Microarray, Gestation

\section{Background}

The Renin-Angiotensin System (RAS) regulates sodium and water homeostasis to maintain blood pressure and fluid balance in all mammals $[1,2]$. The complex integration of actions of circulating maternal RAS in pregnancy plays a key role in pregnancy outcome and maternal health. In human pregnancy, the maternal and fetal circulating RAS interact with various tissue RASs

\footnotetext{
* Correspondence: murray.mitchell@uq.edu.au

${ }^{1}$ Centre for Clinical Diagnostics, University of Queensland Centre for Clinical Research Royal Brisbane and Women's Hospital Campus, Building 71/918,

Royal Brisbane Hospital, Herston, QLD 4029, Australia

Full list of author information is available at the end of the article
}

(ovarian, intrauterine, and intrarenal) [3]. The intrauterine/placental RAS is one of the major extrarenal RAS in pregnancy [4], regulating maternal-fetal blood flow and the uteroplacental blood circulation $[1,5]$. Other uteroplacental RAS functions may include endometrial regeneration, decidualisation, implantation, placentation, uterine contraction, prostaglandin synthesis, and estradiol 17 beta secretion [6, 7]. Because its functions are widespread, dysfunction of the RAS system can lead to complications such as preeclampsia. Placental RAS is itself regulated by several hormones including estrogen and progesterone [8]. 
The RAS pathway begins with the biosynthesis of active enzyme, renin, from its $47 \mathrm{kDa}$ precursor, prorenin [9]. Renin cleaves angiotensinogen (AGT) to form angiotensin, AngI [10]. AngI is then converted to AngII by angiotensin-converting enzyme (ACE) [11, 12]. AngII binds two endogenous receptors: angiotensin II type 1 and 2 receptors (AT1 and AT2) encoded by genes Agtr1 and Agtr2 respectively [2, 4, 13-15]. Agtr1 has two subtypes Agtr1a and Agtr1b. AngII is the primary product of RAS and its activity is terminated by its conversion to Ang 1-7 by MME, membrane metalloendopeptidase [6]. In the RAS pathway, the majority of AngII functions, which include vasoconstriction, vascular permeability and peripheral vascular resistance, stimulation of aldosterone synthesis, angiogenesis and cell growth, are mediated by AT1 [6, 15-17]. In contrast, AT2 is involved in apoptosis, reduction of endothelial cell growth, cell migration, vasodilation, and reduction of vascular injury [2]. AT2 has been hypothesised to antagonize the effects of AT1 $[9,18]$, and hence a distorted balance in the expression of these proteins may be detrimental to pregnancy outcomes. Placental RAS maybe involved in preeclampsia with raised AT1 expression [2]. In human placenta and placental cell lines, the genes involved in the RAS pathway have been detected in both early and late gestation leading to the speculation that it is critical throughout pregnancy [11]. As there is a paucity of data to demonstrate changes to RAS factors change as pregnancy progresses and in complications of pregnancy. Further investigation are required to determine the importance of RAS due to changes in expression and the functional changes associated.

RAS components have been shown to be localised to different regions and cell types of the placenta. AT1 has been mainly localised to human placental syncytiotrophoblasts [2]. The vasodilator ACE2 [1] has been detected in endothelium and syncitium (i.e. multinucleated cells) of labyrinth,, subplacenta (highly vascularised region, part of chorion found between placental disc and basal decidua) and giant cells, in guinea pigs [19]. In late pregnancy, ACE2 is present in the decidual layer and vascular smooth muscles of arteries modulating placental blood flow, regulating vascular remodeling, and potentiating trophoblast invasion and decidualisation earlier in pregnancy [19]. The vasodilator ACE2 has been detected in the endothelium, syncyotiotrophoblast, and cytotrophoblast, in human placenta tissue [20]. It is also present in the intravascular trophoblast and decidual layer and the vascular smooth muscle cells of the umbilical cord vessels [20]. In rats, the upregulation of ACE2 expression in the placenta and uterus contributed to a two-fold increase in total ACE2 activity [21]. Interestingly, although ACE2 expression was upregulated in the rat uterus, it was downregulated at the implantation site [22].
The maternal diet also modulates ACE2 expression; rat dams fed a low protein diet had reduced Ace2 mRNA expression in the placenta leading to fetal growth restriction [1]. Similar results were also reported in ACE2 knockout mice [23]. Overall, ACE2 is involved in modulating placental blood flow, regulating vascular remodeling, and potentiating trophoblast invasion and decidualisation. Moreover the formation of new blood vessels have been affected by the effects of hypoxia in Aminopeptidase-null mice, Aminopeptidase-N, ANPEP being another member of the RAS system [24].

In humans, trophoblast invasion, vascular remodelling and proper placentation are critical to determine pregnancy outcome. Poor vascular remodelling and trophoblast invasion can lead to complications such as preeclampsia. Preeclampsia is associated with short and long term consequences for both the mother and baby including preterm labour and still birth $[14,25,26]$. Alteration of RAS is involved in the pathogenesis of preeclampsia [27] indicating the essential role of RAS for the prevention of pregnancy complications. Gestational age-related studies of RAS throughout early, mid and late gestation are limited. Both systemic and uteroplacental RAS undergo dramatic changes during pregnancy. RAS components in human placenta are expressed from 6 weeks of gestation [12]. Recent studies on the human placenta compared the localisation and expression of RAS in early gestation vs late gestation $[9,18]$. These studies showed that RAS plays a significant role in promoting trophoblast invasion and angiogenesis and is also linked to expression of PTGS2, Prostaglandin-Endoperoxide Synthase 2 (an enzyme potentially involved in the process of parturition).

RAS components have not been comprehensively studied in rat placenta from mid to late gestation. This study specifically aimed to determine gene expression changes of all 17 genes of the RAS pathway in rat placentae. Four time points were chosen as they cover the time period from mid gestation to the day prior to labour onset (E14.25, E15.25, E17.25 and E20). These investigations are apart of a larger gene expression study $[28,29]$. The rat model allows the study of RAS compenents at time-points (mid to late gestation) difficult to be obtained from human and unlike in the mouse, placentation in the rat involves the deep invasion of trophoblast (i.e. a better model of placentation) [30].

\section{Methods}

\section{Animals and diets}

Animal experiments were performed at the Department of Anatomy and Developmental Biology, Monash University (Melbourne, Australia) with the approval of The School of Biomedical Sciences Animal Ethics Committee of the Monash University. Experiments were carried out in accordance with the National Health and Medical 
Research Council of Australia "Australian Code of Practice for the Care and Use of Animals for Scientific Purposes" (7th edition, 2004).

Sprague Dawley dams were used throughout the study. Rats were allowed to adapt to the animal house for one week. Throughout the study, animals were maintained on a diet of standard chow (19.5\% protein, $7 \%$ total fat and $16 \mathrm{MJ} / \mathrm{Kg}$ digestible energy; Glen Forrest StockFeeders WA Rat and Mouse Chow) and water ad libitum prior to diet onset. Rats were maintained in a lightcontrolled environment ( $12 \mathrm{~h}$ light/dark cycle) throughout this study. Female rats were timed mated in a $3 \mathrm{~h}$ window with male Sprague Dawley rats. This was designated as Day 0 of pregnancy. The rationale of using a $3 \mathrm{~h}$ window for mating time is to reduce variability of gestational age among the offspring and to maximize the accuracy in staging of gestation. After mating, dams were housed individually.

\section{Tissue collection}

The formation of the chorioallantoic rat placenta begins on gestational day 12, and therefore the study begins in mid-gestation. Pregnant dams were anaesthetized (Isoflurane Rhodia Australia P/L, VIC, Australia) and humanely killed at embryonic day (E) 14.25, 15.25, 17.25 or 20 ( $n=6$ per gestational age). Whole placentae were collected from the pregnant dams, weighed and then either snap frozen in liquid nitrogen or fixed in $4 \%$ paraformaldehyde prior to processing for immunohistochemistry analysis. Frozen tissues were stored at $-80{ }^{\circ} \mathrm{C}$ until processed and analysed.

\section{RNA isolation}

Rat placental tissues were pulverized into a fine powder using liquid nitrogen and mortar and pestle. Total RNA was extracted from $30 \mathrm{mg}$ of pulverized frozen placental tissue $n=6$ placentae per gestational age group, using the AllPrep DNA/RNA Mini Kit (Qiagen) as per manufacturers' instructions. An on-column Dnase1 treatment step was also carried out in the RNA isolation step to remove any remaining genomic DNA. Following extraction, total RNA was quantified via NanoDrop ND-1000 spectrophotometer (Thermo Scientific, DE, USA). RNA quality was determined with the Agilent 2100 Bioanalyzer (VIC, Australia). RNA samples that fulfilled the following criteria were selected for microarray analysis: (i) $\mathrm{RIN}>8.5$; (ii) $260 / 280$ ratio $>2$; (iii) $260: 230$ ratio $>2.2$. All RNA Integrity Number values were greater than 8.7 .

\section{Microarray analysis using Illumina rat ref arrays}

The microarray analysis (cohort 1) was performed as previously published [28]. In short, $500 \mathrm{ng}$ of total RNA was converted to double stranded cDNA and this was used to generate biotinylated cRNA probes using the Illumina TotalPrep RNA Amplification Kit. Biotin-labelled cRNA were then hybridized to Illumina RatRef-12 Expression BeadChip (San Diego, CA, USA). Slides were scanned on a BeadStation 500 System using Beadscan software version 3.5.31. No RNA samples were pooled in this analysis, each placental samples was analyzed independently. Samples were hybridized into wells at random. The Array experiment readout was deposited on ArrayExpress and includes gene expression data from microarray and high throughput sequencing studies (ArrayExpress Accession number E-MTAB-1987).

RAS genes from the KEGG Renin Angiotensin System pathway were studied for differential expression using SAM (Microarray Software, Stanford University) analyses, whereby differentially expressed placental genes (i.e. $>2$ fold expression change) were identified between each of the 4 gestational age groups.

\section{Quantitative real-time PCR validation}

The RNA was reverse transcribed using the QuantiTect reverse transcription kit (Qiagen) using $1 \mu \mathrm{g}$ of RNA per sample. qPCR (cohort 2) was used to confirm and validate the expression of Ace, Ace2, Thop1, Mme and Anpep in placentae that were different from those used for the microarray analysis. Primers unique for each target gene were designed by Primer-Blast (NCBI), and covered exon-exon junctions. The primer sequences are provided in Table 1.

\section{Immunohistochemistry}

Paraffin sections ( $5 \mu \mathrm{m}, n=3$ sections/gestational age) were deparaffinized in xylene and rehydrated to water through a graded alcohol series. Antigen retrieval was achieved by incubation in citrate buffer $(10 \mathrm{mM}), \mathrm{pH} 6$ at $120{ }^{\circ} \mathrm{C}$ for $30 \mathrm{~s}$ and $90{ }^{\circ} \mathrm{C}$ for $10 \mathrm{~min}$ under higher pressure. Sections were blocked in $\mathrm{MACH}-1$ Background snipper, provided in the MACH-1 ${ }^{\mathrm{Tm}}$ kit (Biocare Medical). Sections were incubated with rabbit anti-CD13 (ANPEP) polyclonal antibody (Abcam Cat no ab108310), prior to incubation with secondary antibodies conjuagated with

Table 1 Primers used for the qPCR experiments for 6 RAS genes

\begin{tabular}{lll}
\hline Gene & Forward $\left(5^{\prime}\right.$-3') & Reverse $\left(5^{\prime}\right.$-3') \\
\hline Ace & ATTGCAGCCGGGCAACTTIT & CGCATTCTCCTCCGTGATGT \\
Ace2 & GAGCCCATATGCCGACCAAA & TCTGCCTCCCCAAAAGGAAC \\
Mme & CCGAAATGACCCAATGCTGC & TGACCAGCTGAATGGCTTCC \\
Thop1 & ATGCTGGAGAACTGGGTGTG & GAAGAGACCTGCATTGGCCT \\
Anpep & AGATTGCCCTGCCTGACTTC & GTTGCCAAACCACTGATGGG \\
Agtr1a & GGATTCGTGGCTTGAGTCCT & TCGAAATCCACTTGACCTGGTG \\
\hline
\end{tabular}


HRP. They were then stained using DAB substrate, whereby positive staining was identified with a brown colour. Purified Rabbit IgG (Sigma Aldrich) was used as a negative isotype control. Sections were counterstained with Mayers hematoxylin and Leica mounting media was used to mount the coverslips. Slides were examined under a microscope (Olympus $\left.{ }^{\mathrm{Tm}}\right)$.

\section{ANPEP western blots}

Protein samples from all four gestational age groups $(n=6)$ were used to examine ANPEP protein expression by Western blotting. All reagents, unless otherwise stated, were purchased from Life Technologies for use in the NuPage Bis-Tris Electrophoresis System. Total protein extracted from rat placental tissue $(30 \mu \mathrm{g})$ were separated in NuPage 4-12 \% Bis-Tris Precast Gels and transferred to a polyvinyl difluoride (PVDF) membrane. Membranes were blocked for $1 \mathrm{~h}$ in blocking solution containing bovine serum albumin powder in Tris Buffered Saline with $0.001 \%$ Tween 20 (Sigma Aldrich, Castle Hill, NSW, Australia). Membranes were incubated overnight at $4{ }^{\circ} \mathrm{C}$ with ANPEP (cd-13, Abcam cat no ab108310) (Santa Cruz Biotechnology) and $\beta$-Actin (Sigma-Aldrich)primary antibodies. Secondary antibodies were purchased from LI-COR ${ }^{\mathrm{mm}}$ Biociences. Odessey ${ }^{\circ} \mathrm{LI}-\mathrm{COR}^{\mathrm{mix}}$ densitometry was used to determine changes in ANPEP (normalized to $\beta$ ACTIN protein) in the placental tissues.

\section{Statistical analysis}

\section{Biolnformatic analyses and statistics}

Microarray bioinformatic analysis was initially performed by using the Illumina Beadstudio and Significance Analysis of Microarry (SAM, Stanford University) software. Data were normalized by performing a probe-intensity transformation and normalization via the Lumi package, Bioconductor. Following normalization, differentially expressed placental genes (i.e. $>2$ fold expression, false discovery rate of $<1 \%$ ) were identified using SAM and were subsequently analyzed using Web-based Gene Set Analysis Toolkit (WebGestalt, http://bioinfo.vanderbilt.edu/ webgestalt/).

Genes were then assigned to their respective functional classes based on the Gene Ontology (GO) database; Renin-Angiotensin KEGG PATHWAY Analysis Differences in group means were assessed by post-hoc comparisons (Bonferroni tests).

For the qPCR and Western blots comparison of the four gestational groups, one-way ANOVA analyses and multiple comparisons were carried out. Correlation plots for both Microarray and qPCR on cohort 1 and 2 respectively were performed using Pearson's Correlation (Additional file 1: Figure S1).

\section{Results}

\section{Differentially expressed RAS genes}

A whole genome microarray was performed [28] to examine differential gene expression in the rat placenta at gestational ages of E14.25, E15.25, E17.25 and E20 (just prior to birth). For this study, genes of only the RAS pathways was specifically analyzed. Genes that demonstrated a $>2$ fold change (microarray analysis) between gestational ages were considered to be differentially expressed (Fig. 1). Six out of 17 RAS genes from the KEGG-RAS signaling pathway (Ace, Ace2, Thop1, Mme, Anpep, and Agtr1a) were differentially expressed (Fig. 2a, c, e, g, i, k). The other 11 genes in the pathway remained unchanged between the 4 gestational age groups (data not shown). The expression of Ace, Ace2, Mme and Agtr1a increased with gestational age whereas, Thoplexpression was decreased with gestational age (Fig. 2). Anpep expression increased with gestational age and then was downregulated just prior to labour onset, hence we investigated this further at the protein level using both Western blots and immunohistochemistry.

\section{Validation of microarray with qPCR}

Microarray results were validated by qPCR by using placenta tissue from an independent cohort of rat dams. Results for the qPCR (cohort 2) are also shown in Fig. 2 for the 6 differentially expressed RAS genes. Additional file 1: Figure S1 shows the correlation between Microarray and qPCR.

\section{Immunohistochemistry on ANPEP}

Anpep gene expression increased from E14.25 to E17.25 and subsequently decreased after E17.25. Immunohistochemistry of ANPEP was performed on paraffin sections of E17.25 and E20 placentae. ANPEP was localized to both junctional and labyrinth zones (Fig. 3). In the junctional zone of E20 placentae, the decidual cells and Giant trophoblast cells had less staining compared to E17.25 placentae. Labyrinth zone localization of ANPEP remained relatively unchanged between E17.25 and E20 placentae.

\section{ANPEP Western blots}

ANPEP protein expression was identified in the placentae at all four gestational ages E14.25, E15.25, E17.25 and E20. However, no significant differences in protein expression were observed within the 4 groups (Fig. 4).

\section{Discussion}

Global RAS gene expression changes in the whole rat placenta throughout the four gestational ages E14.25, E15.25, E17.25 and E20 were studied. Ace, Ace2, Agtr1a (that have previously been studied in rat placenta), and three additional RAS genes Thop1, Anpep and Mme were 


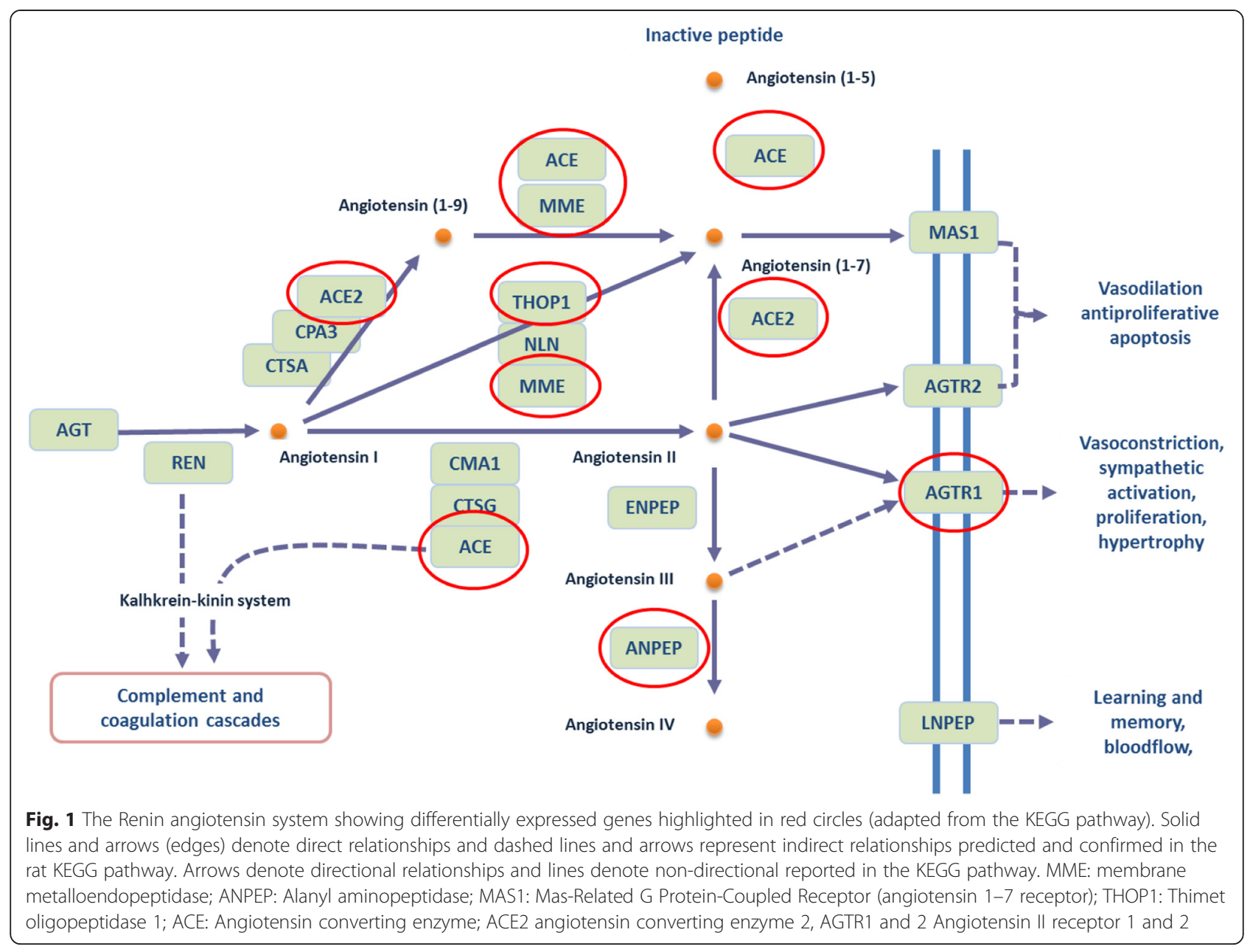

significantly differentially expressed throughout mid to late gestation.

The robustness of the findings in this paper is apparent in that a similar pattern of gene expression of the 6 differentially expressed RAS genes was found in two independent rat cohorts with different techniques (microarray and qPCR, respectively) (Fig. 2). Ace expression was relatively low at E14.25 and E15.25, increased at E17.25 and E20. Similar results were observed for Ace2, Mme, and Agtr1. Consistent with previous studies, these results show that Ace and Ace 2 increase dramatically at late gestation, indicating that the pathway is highly activated at this stage of gestation $[1,22,31]$, so these embryonic days were chosen for study. AngII has previously been shown to be increased with the advancement of pregnancy due to enhanced Ace 2 expression and activity, which correlate with elevated placental-fetal blood flow and rapid fetal growth, especially during mid and late pregnancy [1]. Dietary manipulations may have a significant role in disrupting RAS: RAS function is altered by changes in sodium intake during pregnancy [32], potentially affecting feto-maternal blood flow and hence fetal nutrient supply, fetal growth and birth weight [32]. Interestingly, another study showed that mRNA expression of Ace 2 and Agtr1a was significantly reduced in the labyrinth zone of Sprague Dawley rats fed a low protein diet [1]. Overall, these studies show that elevation of AngII concentration via the expression and activity of ACE and ACE2 and upregulated AT1 expression are potentially required to to increase blood flow in the placenta and therefore may ensure the adequate delivery of nutrients to the fetus, leading to optimal fetal growth.

Mme (membrane metallo-endopeptidase) is an enzyme that inactivates RAS by converting AngI to Angiotensin (1-7). Interestingly, MAS1 receptor gene, Mas-Related G Protein-Coupled Receptor (angiotensin 1-7 receptor) expression remained unchanged (Fig. 1). Mme has been studied in renal, lung and neural tissues, however, very little is known about its role in the placenta [33]. SNPs leading to truncations of the Mme gene and the loss of neutral endopeptidase (NEP) protein are theorised to be the cause for allo-immunisation during pregnancy [34]. Studies in rat brain and neural tissue as well as in human endometrium show that hormones can regulate 


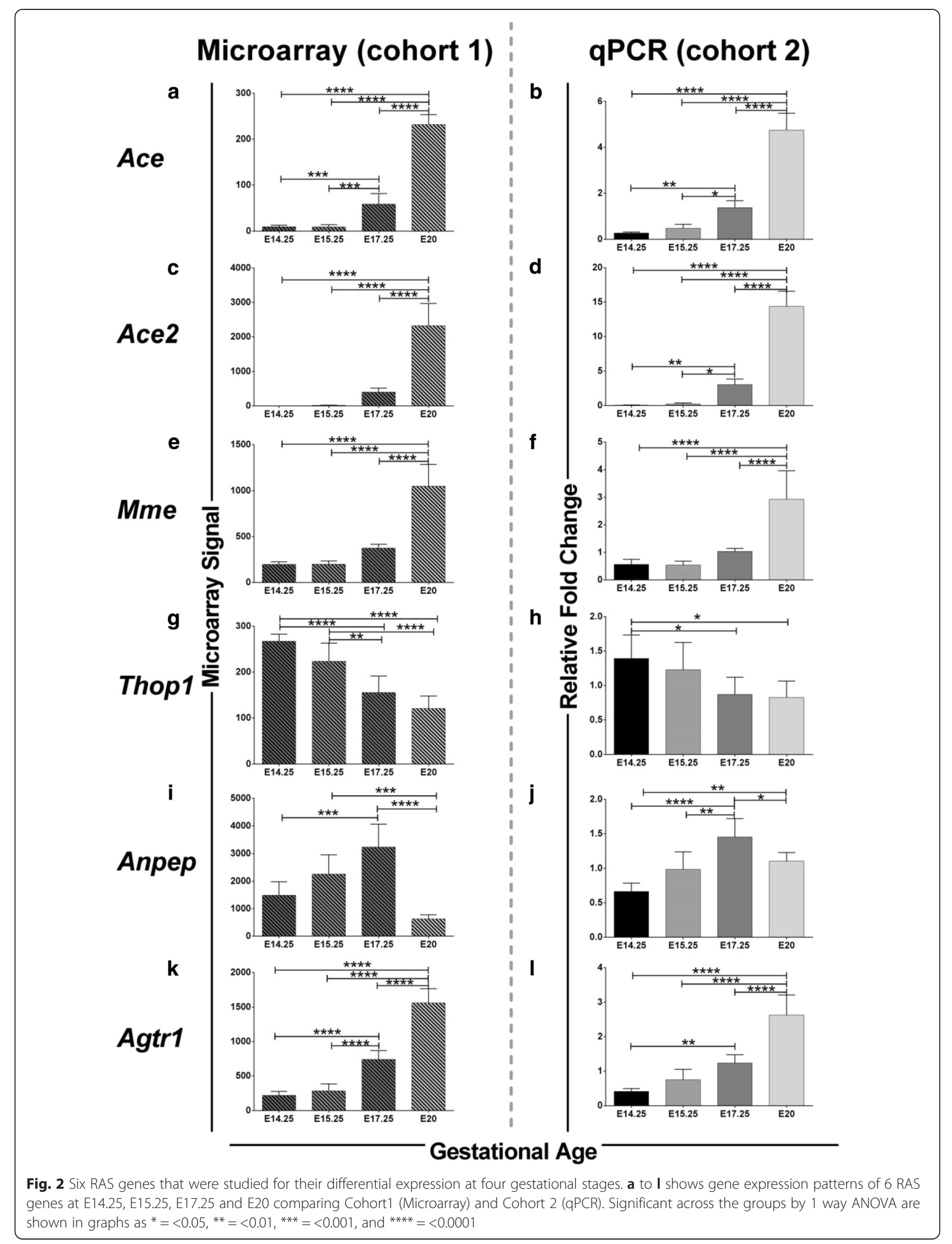




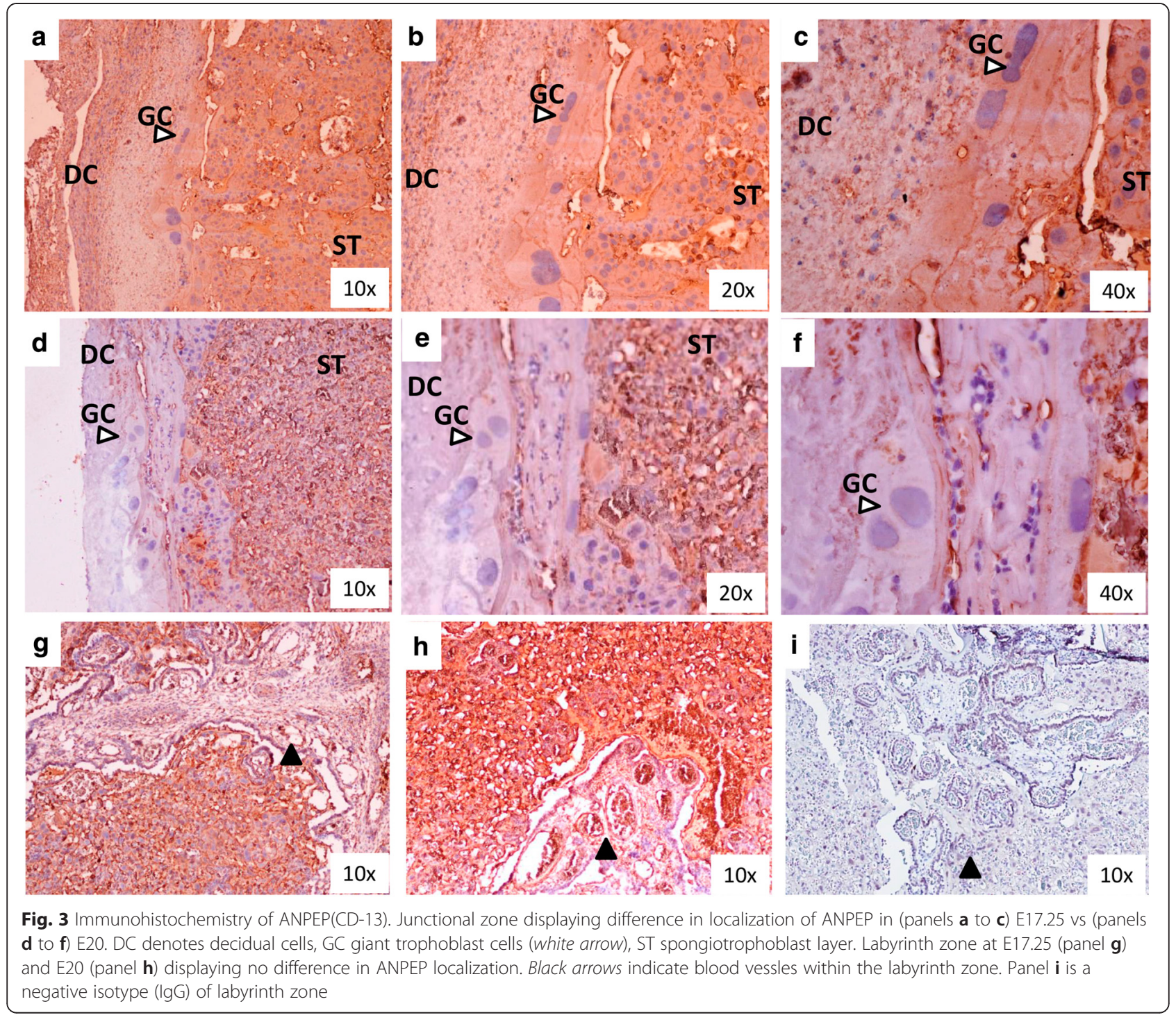

Mme expression [35, 36]. Since MME enzyme is an important player in several tissues and in the RAS pathway, it is important to study its expression and function in placenta and pregnancy to elucidate its link to pregnancy complications.

The results of this study showed that the expression of another novel gene, Thop1, gradually decreases over time during pregnancy (from E14.25 to E15.25 and further decreases at E17.25) and is relatively low at E20 prior to labour onset. There is a paucity of data on Thop1 expression and function in the placenta, however in the brain, a therapeutic potential as an anti-tumour agent has recently been reported $[37,38]$.

ANPEP (also known as APN) is an enzyme that selectively hydrolyses $\mathrm{N}$ terminal acidic amino acids such as glutamyl and aspartyl residues from peptide or substrate, and can degrade ANGII to ANGIII in the RAS pathway. It has also been previously established that ANPEP is localised to trophoblast, fetal arterioles and venules in the stem villi of the human placenta. The mRNA, protein and the activity of ANPEP is known to be higher in human placentae of pregnancies complicated with preeclampsia compared to normal pregnacy [39]. In APN-null mice, investigations on the effect of hypoxic conditions found marked reductions in formations of new blood vessels (compared to WT animals), indicating the importance of this factor in angiogenesis [24]. In the current study, Anpep gene expression was significantly lower at E20 (just prior to labour onset). We investigated further the protein expression of ANPEP to examine if the differences at the gene expression were detectable at the protein level. Protein expression of ANPEP (Western blot on whole placental tissue) did not significantly differ between the four groups. However, ANPEP localization by immunohistocheisty identified staing differences in E17.25 and E20 placentae, as E17.25 ANPEP localization was identified in 


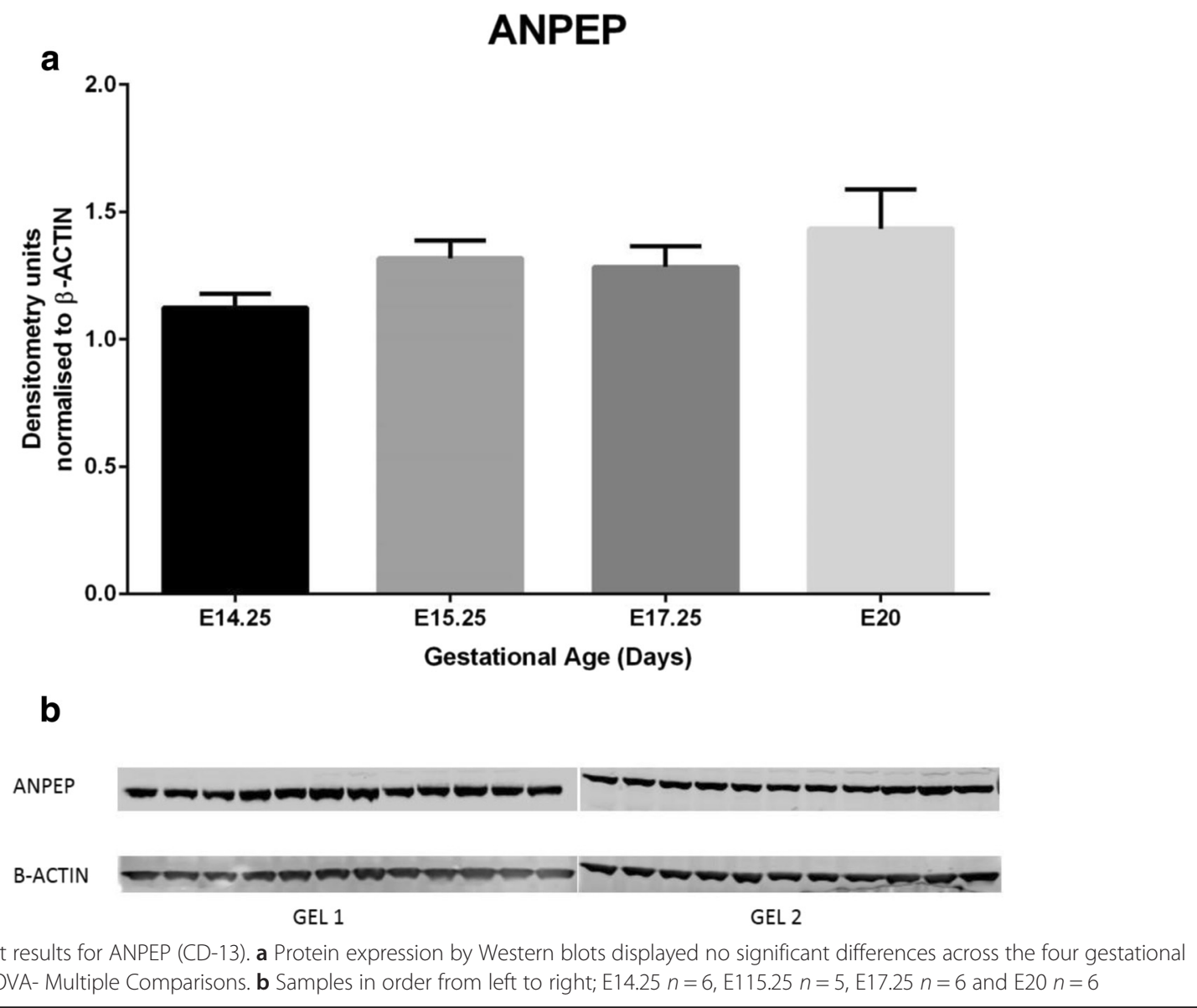

the decidual layer, giant trophoblast cells and the spongiotrophoblast layer whereasat E20 staining was only visible in spongiotrophoblast layer (no staining in the decidual layer and giant cells). No visual differences in the localization of ANPEP was seen in labyrinth zone of the rat placentae. In future studies, differences in the protein expression by Western blot of ANPEP and other members of the RAS pathway may need to be investigated in separated junctional and labyrinth zones to fully elucidate changes to the protein expressions. Furthermore, immunohistochemical studies (such as those described in this study) may provide more extensive detail as to the cell types expressing members of the RAS pathway. The finding of these studies mayallow for more specific investigations to be performed to the role of RAS pathway members in angiogenesis as well as the overall importance of this pathway in labour. These finding may also lead to the development of functional studies ulitising knockout models in animal models (e.g. mice) of the relavent RAS factors/pathway members.

The expression of 11 RAS genes whose expression remained unchanged throughout the four gestational groups were Agt, Ren, Ctsa, Cpa3, Nln, Cma1, Ctsg,
Enpep, Mas, Agtr2 and Lnpep (data not shown). Further studies will be required to study these genes at the protein level and also comparing several regions of the rat placenta.

Many studies have indicated that RAS dysfunction is associated withpregnancy complications such as preeclampsia [25]. Normal placentataion involves trophoblast invasion of maternal decidua and remodelling of spiral arteries (replacement of smooth muscle cell layer with fibrinoid material) which causes vessels to increase in diameter allowing for increased blood flow into the placenta [40]. Abnormal placentation can lead to pregnancy complications such as preeclamsia. Preeclampsia is associated with shallow trophoblast invasion of the maternal spiral arteries in the endometrium of the uterus, leading to improper remodelling of the uterine spiral arteries $[4,31,41]$. In preeclampsia, increased sensitivity to Ang II (important in blood flow maintenance) has been documented and this phenomenon may be developed before clinical manifestations of the diesease [25]. Women with preeclampsia have been seen to have lower circulating levels of RAS factors, however, AngII levels, angiotensinogen and Agtr1 receptor mRNA is increased 
in placentae of preeclamptic women [41]. Further investigations are required in normal and complicated pregnancies to fully elucidate the role of RAS in maintaining a healthy pregnancy or contributing to pregnancy complications.

\section{Conclusions}

Adequate blood flow in the placenta is vital for the maintenance of placental function, development and fetal growth, maintenance and development. The intrauterine/ placental RAS is important in pregnancy, regulating maternal-fetal blood flow and the uteroplacental blood circulation. The current study investigated the expression of members of the RAS pathway in relatively normal rat placentae at different gestational ages. Six of the 17 RAS genes were differentially expressed in the rat placenta of 4 different gestational age groups by microarray and the differernatial expressions validated by qPCR. Immunohistochemical analysis of ANPEP identified differences in localization at E20 compared to E17.25 rat placentae. These findings present the need for further comprehensive investigations of RAS genes, specific placental localizations and functional studies in normal and complicated pregnancies.

\section{Additional file}

Additional file 1: Figure S1. Correlation plots Microarray Signal vs qPCR gene expression. A) to F) displays correlation plots for Ace, Ace2, Mme, Thop1, Anpep, Agtrla. All the results were significant using Pearson Correlation. (DOCX $59 \mathrm{~kb})$

\section{Competing interests}

The authors declare that they have no competing interests.

\section{Authors' contibutions}

JA, RJW, GER designed the animal study. JA. RJW conducted the animal studies, sample collection. KV, HC carried out the gene expression studies. PV, HP performed immunohistochemical studies. MDN, GER, HC, KV performed analyses. KV, HP MDM drafted the manuscript. All authors reviewed manuscript and MDM approved the final manuscript for submission. All authors read and approved the final manuscript.

\section{Acknowledgements}

Microarray hybridization analysis was performed by Mr. Nick Matigian (Eskitis Institute for Cell and Molecular Therapies, Griffith University, Queensland, Australia). MDM received funding from Therapeutic Innovation Australia -Queensland Node.

\footnotetext{
Author details

${ }^{1}$ Centre for Clinical Diagnostics, University of Queensland Centre for Clinical Research Royal Brisbane and Women's Hospital Campus, Building 71/918, Royal Brisbane Hospital, Herston, QLD 4029, Australia. ${ }^{2}$ Department of Anatomy \& Developmental Biology Monash University, Clayton, VIC 3800, Australia. ${ }^{3}$ School of Medicine (Optometry), Deakin University, Pigdons Road, Waurn Ponds, VIC 3800, Australia.
}

Received: 28 May 2015 Accepted: 3 August 2015

Published online: 12 August 2015

\section{References}

1. Gao H, Yallampalli U, Yallampalli C. Maternal protein restriction reduces expression of angiotensin 1-converting enzyme 2 in rat placental labyrinth zone in late pregnancy. Biol Reprod. 2012;86(2):31. doi:10.1095/biolreprod.111.094607.

2. Mistry HD, Kurlak LO, Broughton PF. The placental renin-angiotensin system and oxidative stress in pre-eclampsia. Placenta. 2013;34(2):182-6. doi:10.1016/j.placenta.2012.11.027.

3. Lumbers ER, Pringle KG. The roles of circulating renin-angiotensinaldosterone system in human pregnancy. Am J Physiol Regul Integr Comp Physiol. 2013. doi:10.1152/ajpregu.00034.2013.

4. Irani RA, Xia Y. The functional role of the renin-angiotensin system in pregnancy and preeclampsia. Placenta. 2008;29(9):763-71. doi:10.1016/j.placenta.2008.06.011.

5. Cooper AC, Robinson G, Vinson GP, Cheung WT, Broughton PF. The localization and expression of the renin-angiotensin system in the human placenta throughout pregnancy. Placenta. 1999;20(5-6):467-74. doi:10.1053/plac.1999.0404.

6. Goyal R, Lister R, Leitzke A, Goyal D, Gheorghe CP, Longo LD. Antenatal maternal hypoxic stress: adaptations of the placental renin-angiotensin system in the mouse. Placenta. 2011;32(2):134-9. doi:10.1016/j.placenta.2010.11.004.

7. Hagemann A. Solution of methodological problems in prorenin measurement and investigations of tissue renin-angiotensin systems in the female reproductive tract. Dan Med Bull. 1997;44(5):486-98.

8. Pan N, Frome WL, Dart RA, Tewksbury D, Luo J. Expression of the renin-angiotensin system in a human placental cell line. Clin Med Res. 2013;11(1):1-6. doi:10.3121/cmr.2012.1094.

9. Pringle KG, Zakar T, Yates D, Mitchell CM, Hirst JJ, Lumbers ER. Molecular evidence of a (pro)renin/ (pro)renin receptor system in human intrauterine tissues in pregnancy and its association with PGHS-2.J Renin Angiotensin Aldosterone Syst. 2011;12(3):304-10. doi:10.1177/1470320310376554.

10. Morgan L, Broughton PF, Kalsheker N. Angiotensinogen: molecular biology, biochemistry and physiology. Int J Biochem Cell Biol. 1996;28(11):1211-22.

11. Wang Y, Pringle KG, Lumbers ER. The effects of cyclic AMP, sex steroids and global hypomethylation on the expression of genes controlling the activity of the renin-angiotensin system in placental cell lines. Placenta. 2013;34(3):275-80. doi:10.1016/j.placenta.2012.12.018.

12. Schwentner $L$, Wockel $A$, Herr D, Wulff $C$. Is there a role of the local tissue RAS in the regulation of physiologic and pathophysiologic conditions in the reproductive tract? J Renin Angiotensin Aldosterone Syst. 2011;12(4):385-93. doi:10.1177/1470320311418140.

13. Bedard S, Sicotte B, St-Louis J, Brochu M. Modulation of body fluids and angiotensin II receptors in a rat model of intra-uterine growth restriction. J Physiol. 2005;562(Pt 3):937-50. doi:10.1113/jphysiol.2004.064683.

14. Takeda-Matsubara Y, Iwai M, Cui TX, Shiuchi T, Liu HW, Okumura M, et al. Roles of angiotensin type 1 and 2 receptors in pregnancyassociated blood pressure change. Am J Hypertens. 2004;17(8):684-9. doi:10.1016/j.amjhyper.2004.03.680.

15. Williams PJ, Mistry HD, Innes BA, Bulmer JN, Broughton PF. Expression of AT1R, AT2R and AT4R and their roles in extravillous trophoblast invasion in the human. Placenta. 2010;31(5):448-55. doi:10.1016/j.placenta.2010.02.014.

16. Ishimaru T, Ishida J, Nakamura S, Hashimoto M, Matsukura T, Nakamura A, et al. Short-term suppression of the renin-angiotensin system in mice associated with hypertension during pregnancy. Mol Med Rep. 2012;6(1):28-32. doi:10.3892/mmr.2012.886

17. Squires PM, Kennedy TG. Evidence for a role for a uterine renin-angiotensin system in decidualization in rats. J Reprod Fertil. 1992;95(3):791-802.

18. Pringle KG, Tadros MA, Callister RJ, Lumbers ER. The expression and localization of the human placental prorenin/renin-angiotensin system throughout pregnancy: roles in trophoblast invasion and angiogenesis? Placenta. 2011;32(12):956-62. doi:10.1016/j.placenta.2011.09.020.

19. Valdes G, Corthorn J, Bharadwaj MS, Joyner J, Schneider D, Brosnihan KB. Utero-placental expression of angiotensin-(1-7) and ACE2 in the pregnant guinea-pig. Reprod Biol Endocrinol. 2013;11:5. doi:10.1186/1477-7827-11-5.

20. Valdes G, Neves LA, Anton L, Corthorn J, Chacon C, Germain AM, et al. Distribution of angiotensin-(1-7) and ACE2 in human placentas of normal and pathological pregnancies. Placenta. 2006;27(2-3):200-7. doi:10.1016/j.placenta.2005.02.015.

21. Levy A, Yagil Y, Bursztyn M, Barkalifa R, Scharf S, Yagil C. ACE2 expression and activity are enhanced during pregnancy. Am J Physiol Regul Integr Comp Physiol. 2008;295(6):R1953-61. doi:10.1152/ajpregu.90592.2008.

22. Neves $L A$, Stovall $K$, Joyner J, Valdes $G$, Gallagher PE, Ferrario CM, et al. ACE2 and ANG-(1-7) in the rat uterus during early and late gestation. Am J Physiol Regul Integr Comp Physiol. 2008;294(1):R151-61. doi:10.1152/ajpregu.00514.2007. 
23. Bharadwaj MS, Strawn WB, Groban L, Yamaleyeva LM, Chappell MC, Horta C, et al. Angiotensin-converting enzyme 2 deficiency is associated with impaired gestational weight gain and fetal growth restriction. Hypertension. 2011;58(5):852-8. doi:10.1161/HYPERTENSIONAHA.111.179358.

24. Rangel R, Sun Y, Guzman-Rojas L, Ozawa MG, Sun J, Giordano RJ, et al. Impaired angiogenesis in aminopeptidase N-null mice. Proc Natl Acad Sci U S A. 2007;104(11):4588-93. doi:10.1073/pnas.0611653104.

25. Uddin MN, Agunanne E, Horvat D, Puschett JB. Alterations in the renin-angiotensin system in a rat model of human preeclampsia. Am J Nephrol. 2010;31(2):171-7. doi:10.1159/000267099.

26. Genest DS, Falcao S, Michel C, Kajla S, Germano MF, Lacasse AA, et al. Novel Role of the Renin-Angiotensin System in Preeclampsia Superimposed on Chronic Hypertension and the Effects of Exercise in a Mouse Model. Hypertension. 2013. doi:10.1161/HYPERTENSIONAHA.113.01983.

27. Rodriguez M, Moreno J, Hasbun J. RAS in Pregnancy and Preeclampsia and Eclampsia. Int J Hypertens. 2012;2012:739274. doi:10.1155/2012/739274.

28. Vaswani K, Hum MW, Chan HW, Ryan J, Wood-Bradley RJ, Nitert MD, et al. The effect of gestational age on angiogenic gene expression in the rat placenta. PloS one. 2013;8(12), e83762. doi:10.1371/journal.pone.0083762.

29. Dekker Nitert M, Vaswani K, Hum M, Chan HW, Wood-Bradley R, Henry S, et al. Maternal high-fat diet alters expression of pathways of growth, blood supply and arachidonic acid in rat placenta. J Nutr Sci. 2013;2, e41. doi:10.1017/jns.2013.36

30. Soares MJ, Chakraborty D, Karim Rumi MA, Konno T, Renaud SJ. Rat placentation: an experimental model for investigating the hemochorial maternal-fetal interface. Placenta. 2012;33(4):233-43. doi:10.1016/j.placenta.2011.11.026.

31. Brosnihan KB, Hering $L$, Dechend $R$, Chappell MC, Herse F. Increased angiotensin $I 1$ in the mesometrial triangle of a transgenic rat model of preeclampsia. Hypertension. 2010;55(2):562-6. doi:10.1161/HYPERTENSIONAHA.109.145656.

32. Leandro SM, Furukawa LN, Shimizu MH, Casarini DE, Seguro AC, Patriarca G, et al. Low birth weight in response to salt restriction during pregnancy is not due to alterations in uterine-placental blood flow or the placental and peripheral renin-angiotensin system. Physiol Behav. 2008;95(1-2):145-51. doi:10.1016/j.physbeh.2008.05.011.

33. de Gortari P, Vargas MA, Martinez A, Garcia-Vazquez Al, Uribe RM, Chavez-Gutierrez L, et al. Stage-specific modulation of neprilysin and aminopeptidase $\mathrm{N}$ in the limbic system during kindling progression. J Mol Neurosci. 2007;33(3):252-61. doi:10.1007/s12031-007-0020-9.

34. Debiec $H$, Nauta J, Coulet F, van der Burg M, Guigonis V, Schurmans T, et al. Role of truncating mutations in MME gene in fetomaternal alloimmunisation and antenatal glomerulopathies. Lancet. 2004;364(9441):1252-9. doi:10.1016/S0140-6736(04)17142-0.

35. Yao M, Nguyen TV, Rosario ER, Ramsden M, Pike CJ. Androgens regulate neprilysin expression: role in reducing beta-amyloid levels. J Neurochem. 2008;105(6):2477-88. doi:10.1111/j.1471-4159.2008.05341.x.

36. Li C, Booze RM, Hersh LB. Tissue-specific expression of rat neutral endopeptidase (neprilysin) mRNAs. J Biol Chem. 1995;270(11):5723-8.

37. Qi L, Li SH, Si LB, Lu M, Tian H. Expression of THOP1 and its relationship to prognosis in non-small cell lung cancer. PloS one. 2014;9(9), e106665. doi:10.1371/journal.pone.0106665.

38. Massarelli EE, Casatti CA, Kato A, Camargo AC, Bauer JA, Glucksman MJ, et al. Differential subcellular distribution of neurolysin (EC 3.4.24.16) and thimet oligopeptidase (EC 3.4.24.15) in the rat brain. Brain Res. 1999;851(1-2):261-5.

39. Itakura A, Mizutani S. Involvement of placental peptidases associated with renin-angiotensin systems in preeclampsia. Biochim Biophys Acta. 2005;1751(1):68-72. doi:10.1016/j.bbapap.2005.03.001.

40. Hering L, Herse F, Geusens N, Verlohren S, Wenzel K, Staff AC, et al. Effects of circulating and local uteroplacental angiotensin II in rat pregnancy. Hypertension. 2010;56(2):311-8. doi:10.1161/HYPERTENSIONAHA.110.150961.

41. Anton L, Merrill DC, Neves LA, Diz DI, Corthorn J, Valdes G, et al. The uterine placental bed Renin-Angiotensin system in normal and preeclamptic pregnancy. Endocrinology. 2009;150(9):4316-25. doi:10.1210/en.2009-0076.

\section{Submit your next manuscript to BioMed Central and take full advantage of:}

- Convenient online submission

- Thorough peer review

- No space constraints or color figure charges

- Immediate publication on acceptance

- Inclusion in PubMed, CAS, Scopus and Google Scholar

- Research which is freely available for redistribution

Submit your manuscript at www.biomedcentral.com/submit 